\title{
The muscle findings in the neuroleptic malignant syndrome associated with lysergic acid diethylamide
}

\author{
W M H Behan, A M O Bakheit, P O Behan, I A R More PhD
}

\begin{abstract}
A detailed pathological description of the muscle findings in a case of the neuroleptic malignant syndrome (NMS) following ingestion of lysergic acid diethylamide (LSD) is given, including the first ultrastructural analysis. Focal necrosis, oedema, and hypercontraction of fibres with glycogen and lipid depletion, were identified, all of which had resolved completely a year later. The findings are compared with those in malignant hyperthermia. It is suggested that the results support the view that in NMS, the muscle rigidity is due to central mechanisms and, in both this disorder and malignant hyperthermia, it is responsible for the hyperpyrexia and its life-threatening complications.
\end{abstract}

The neuroleptic malignant syndrome (NMS) is a rare, but almost certainly underdiagnosed, complication of neuroleptic therapy, occurring in from $0.5 \%$ to $1 \%$ of treated patients and associated with a mortality rate of approximately $20 \% .^{1}$ Numerous antipsychotic drugs have been shown to precipitate NMS, as may abrupt discontinuation of anti-Parkinsonian agents. ${ }^{2}$ Cocaine has also been implicated ${ }^{3}$ and we have recently seen a case associated with lysergic acid diethylamide (LSD). The disorder is characterised by a high fever, extrapyramidal rigidity, altered consciousness and increased creatine kinase concentrations. ${ }^{1}$ Its clinical features are similar to those found in malignant hyperthermia $(\mathrm{MH})^{4}$ but whereas the muscle biopsy findings have been carefully studied in the latter, ${ }^{5}$ they have not been commented on, apart from one brief report, ${ }^{6}$ in NMS. We report the features in our case, including the results of a detailed examination of the muscle, and compare the findings in both conditions.

\section{Case report}

A 21 year old man was admitted unconscious to hospital, having consumed a large amount of alcohol and one tablet of lysergic acid diethylamide (LSD) a few hours previously. There was no significant family or past medical history. He was known to have smoked cannabis in the past but had not used any other drugs. On admission he was unconscious, but could localise pain. His temperature was $37 \cdot 5^{\circ} \mathrm{C}$. There was severe bilateral extrapyramidal rigidity with bilateral extensor plantar responses. The rest of the neurological and general physical examination was normal. The following day his conscious level improved spontaneously but he developed transient visual hallucinations. He was not confused but his speech was slurred and he was profoundly hypokinetic. Over the next five days his level of consciousness fluctuated rapidly and considerably, sometimes with the deterioration lasting several hours. At the same time, he continued to have an intermittent low grade pyrexia. Dantrolene sodium therapy $25 \mathrm{mg}$ three times daily produced an immediate, excellent response and he recovered completely in ten days.

\section{Laboratory findings}

His haemoglobin was $15 \mathrm{~g} / \mathrm{dl}$, with a peripheral white blood cell count of $10 \times 10^{9} / 1$. The ESR was $16 \mathrm{~mm}$ in the first hour. Serum urea, electrolytes and creatinine were normal as were the blood glucose and arterial blood gases. Aspartate aminotransferase was $126 \mathrm{IU} / 1$ and lactate dehydrogenase was $727 \mathrm{IU} / 1$ (normal values for our laboratory are $10-40$ and 240-525 respectively). Serum creatine phosphokinase was $807 \mathrm{IU} / 1$ on admission, rising to $3090 \mathrm{IU} / 1$ after 10 days (normal $150 \mathrm{IU} / 1$ ). Urine and repeated cultures were sterile. The CSF was normal with normal pressure. Skull radiographs, an EEG, CT and MRI brain scans revealed no abnormality. The serum concentration of LSD was 69.9 nanogrammes/ml 24 hours after admission but tests for other drugs were negative. Urinary myoglobin was not detected.

\section{Muscle biopsy findings}

Three needle cores of skeletal muscle were taken from the right vastus lateralis on the day of admission, orientated transversely using a dissecting microscope and then fixed in thiopentane cooled with liquid nitrogen. Two tiny fresh fragments were fixed in $2 \%$ glutaraldehyde and processed for electron microscopic examination. Frozen tissue sections were cut at $6 \mu$ in the cryostat and stained with haematoxylin and eosin, Gomori's trichrome, adenosine triphosphatase at $\mathrm{pH} 4.3$ and $\mathbf{9 \cdot 4}$, succinate dehydrogenase, NADH- 


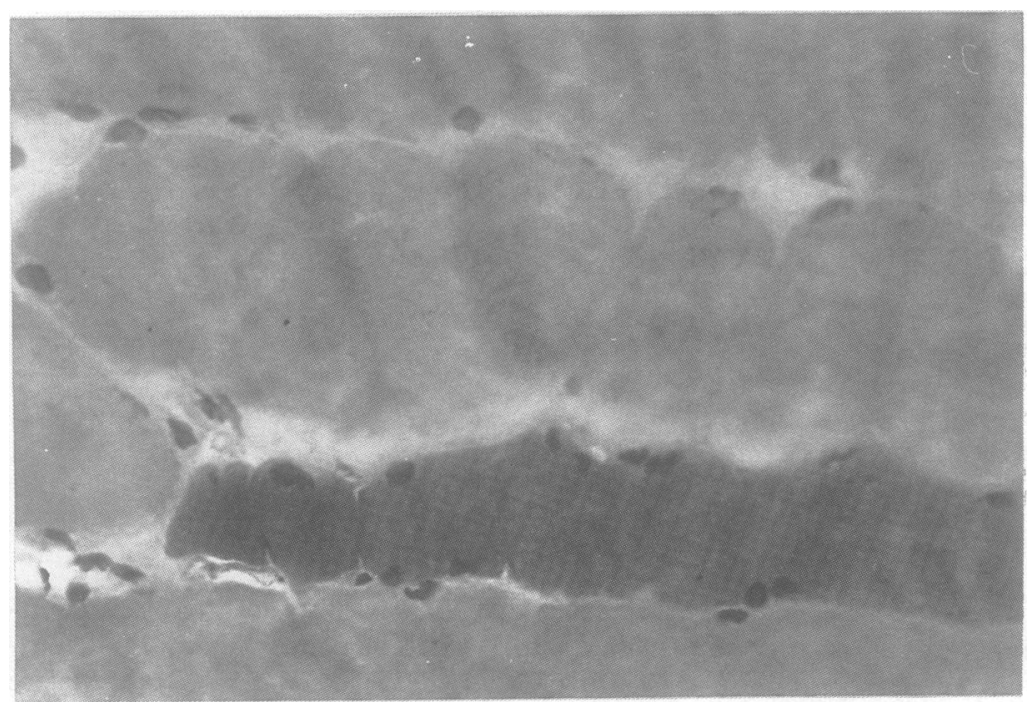

Figure 1 Early hyaline necrosis and peripheral phagocytosis of fibre. $R$ vastus lateralis. Gomori trichrome $\times 400$. dilated sarcoplasmic reticulum or inclusion bodies were present.

One year later the patient was rebiopsied, samples being taken from the left vastus lateralis. Specimens processed in an identical manner were completely normal.

\section{Discussion}

The case described here is typical of NMS, with extrapyramidal rigidity, pyrexia, a fluctuating level of consciousness and an increased serum creatine phosphokinase concentration, lasting for several days. The patient also had increased liver enzymes, a common finding in NMS. The only drug he had taken was LSD and its presence was confirmed in a blood test taken 24 hours after admission. Thus it appears that LSD precipitated the syndrome in this young man. Its effect may have been potentiated by dehydration due to excessive alcohol intake.

The muscle biopsy revealed evidence of recent focal necrosis and generalised oedema. An intense irregular contraction of the fibres

tetrazolium reductase, alkaline phosphatase, periodic acid schiff (with and without diastase) and oil red 0 for lipid, as described. ${ }^{7}$

On microscopy, there was mild generalised oedema. Occasional fibres showed early evidence of necrosis, with a hyaline appearance and infiltration by macrophages (fig 1). In addition, an unusual feature was the appearance, most obvious on the $\mathrm{H} \& \mathrm{E}$ and Gomori stains, of irregularly "scalloped fibres" showing a dense refractile rim (fig 2). Short, irregular contraction bands could also be seen easily in most fibres on transverse and longitudinal section (fig 2). Histochemical stains revealed moderate glycogen and lipid depletion, especially in Type 1 fibres but no other abnormalities. Electron microscopy showed complete fragmentation or disappearance of the $Z$ lines in some fibres and streaming and dispersal of band material in others. No ruptured mitochondria, membranous bodies, increased sarcoplasmic fluid,

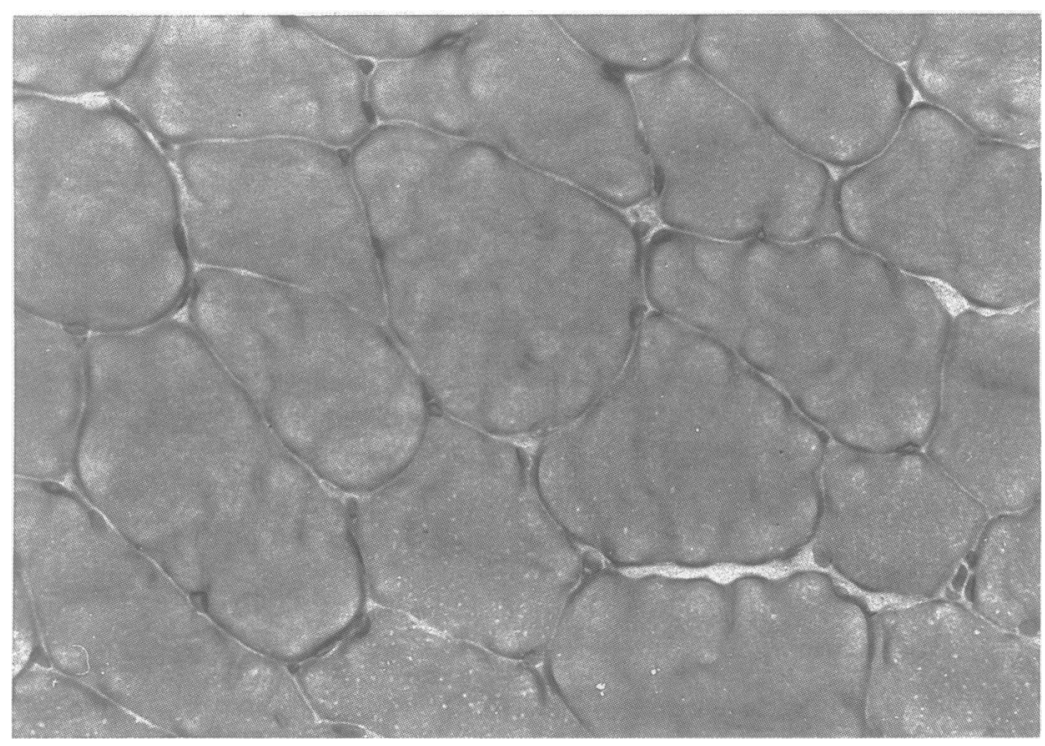

Figure 2 Irregularly "scalloped" fibres with short, broken contraction bands. $H \in E \times 400$. was also visible, confirmed on ultrastructure.

This change can be an artefact due to poor processing but the tissue for frozen studies and for electron microscopy had been processed in the usual manner, according to established methods ${ }^{7}$ and other specimens processed at the same time, did not show any contractions. In addition, when a rebiopsy was taken one year later, the fibres were completely normal. In summary therefore there was obvious damage to the fibres in this patient with myodema, focal necrosis, glycogen and lipid depletion. Severe,

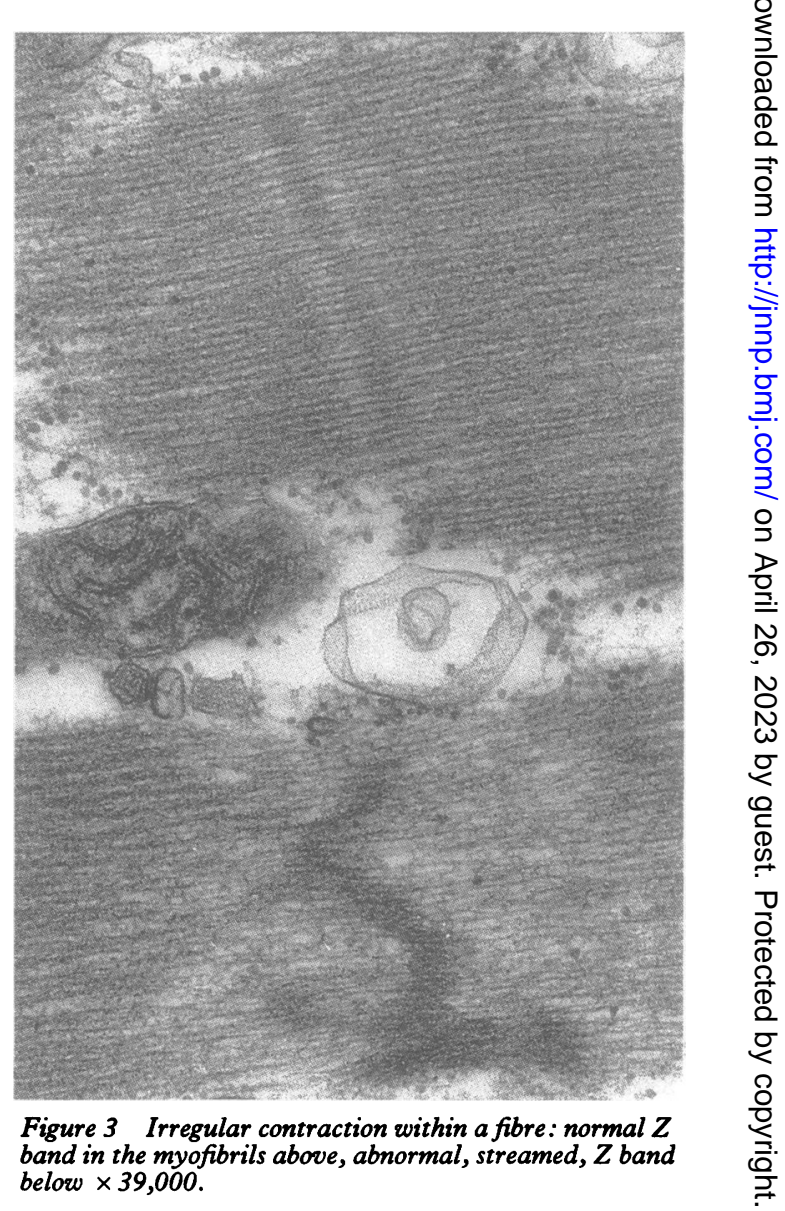


irregular contraction had also occurred. No evidence of any pre-existing muscle disease was present.

There is only one report of the detailed muscle pathology in NMS and that does not include ultramicroscopy. Martin and Swash ${ }^{6}$ examined a biopsy taken one hour after death in a patient who died of the syndrome after a three day history. They reported acute muscle necrosis with oedema and conspicuous glycogen depletion, findings similar to ours. They did not observe the same hypercontraction but this may have been because the biopsy was taken after three days, and after death. Because they did not find lipid depletion they considered that there was uncoupling of muscle metabolism and suggested that metabolic muscle damage had led to excessive muscle activity and rigidity, resulting in hyperthermia. They thought the neuroleptic drug produced the associated extrapyramidal signs, by a different mechanism.

We disagree with the concept that the drug produces primarily a myopathic effect and this leads to the hyperthermia. We think that a reverse mechanism is occurring; in other words, the muscle damage is itself due to central nervous system mechanisms which cause excessive and continuous muscle contraction, and hyperpyrexia. A vicious circle then ensues with the hyperpyrexia causing more muscle damage, and the sustained muscle activity increasing the body temperature. Our view is supported by a recent careful, neuropathological study of a patient who died of NMS. ${ }^{8}$ Localised necrosis of the anterior hypothalamus was found, the area which has been shown experimentally to play a major role in temperature regulation. The authors suggested that damage to neurons in this area could lead to uninhibited release of neurotransmitters and an adverse effect on the adjacent centres of thermoregulation. ${ }^{8}$

It is important to compare the biopsy findings in MNS with those in $M H$, since each condition is associated with muscle rigidity and hyperpyrexia ${ }^{24}$ following drug (or anaesthetic) administration. Harriman carried out an exhaustive survey of muscle in the latter condition, personally examining a total of 1400 biopsies and reviewing all the world literature. The majority of specimens had no evidence of muscle damage; when abnormalities were present, they were non-specific, consisting of occasional central nuclei, moth-eaten fibres and cores. Interestingly enough, however, some cases had hypercontraction of fibres and Z-band streaming, like the case we report here. Harriman considered, as we do in NMS, that the contraction went on to produce fibre necrosis and not the other way round. ${ }^{5}$ Indeed, ultrastructural studies of $\mathrm{MH}$ now support his view, in suggesting that non-specific muscle damage follows the excessive contraction, and perhaps, the hyperpyrexia. ${ }^{11}$
Malignant hyperthermia of course, has a genetic basis with an autosomal dominant inheritance and is considered to be due to defective membrane regulation of calcium in the presence of certain anaesthetic agents. ${ }^{2}$ Although there are no reports of NMS in siblings of affected cases, Caroff et al ${ }^{9}$ have found that in vitro exposure to halothane of muscle fibres from patients who have recovered from NMS, shows the same response as patients susceptible to $\mathrm{MH}$. On the other hand, neuroleptic drugs have been reported to attenuate, or even prevent, the development of $\mathrm{MH}$ in its experimental model, in susceptible pigs. ${ }^{10}$

Both NMS and MH respond to dantrolene, a specific muscle relaxant with no action on the central nervous system, smooth or cardiac muscle. ${ }^{12}$ It has been shown in NMS that the fall in temperature associated with dantrolene coincides with muscle relaxation. ${ }^{13}$ Dantrolene sodium brought about a dramatic response in our patient: a few hours later, his high temperature and muscle rigidity had subsided and his level of consciousness improved.

In NMS, there is evidence of central nervous damage, with structural lesions in the thermoregulatory centre, ${ }^{8}$ accompanied by increased urinary excretion of dopamine metabolites $^{14}$ and a favourable response to bromocriptine.$^{15} \mathrm{We}$ postulate that this damage first causes extrapyramidal rigidity and that the sustained tonic muscular contraction results in thermogenesis. Thus muscle rigidity is the final common component in NMS and $\mathrm{MH}$ and, unless successfully treated, for example, by dantrolene, will compound the hyperpyrexia and its life-threatening complications.

1 Gibb WRG, Lees AJ. The neuroleptic malignant syndrome

Quart $J$ Med 1985;56:421-9.
2 Guze BH, Baxter LR. Neuroleptic malignant syndrome. Guze BH, Baxter LR. Neuroleptic
New Engl J Med 1985;313:163-6.

3 Kosten TR, Kleber HD. Sudden death in cocaine abusers: relation to neuroleptic malignant syndrome. Lancet 1987;i:1198-9.

4 Gronert GA. Malignant hyperthermia. Anaesthesiology 1980;53:395-423.

5 Harriman DGF. Malignant hyperthermia myopathy-a critical review. Br J Anaesth 1988;60:309-16.

6 Martin D, Swash M. Muscle pathology in the neuroleptic malignant syndrome. J Neurol 1987;235:120-1.

7 Dubowitz V. Muscle biopsy. A practical approach, 2nd ed. London: Bailliere Tindall, 1985:19-40.

8 Horn E, Lach B, Lapierre Y, et al. Hypothalamic pathology in the neuroleptic malignant syndrome. Am J Psychiatry 1988;145:617-20.

9 Caroff SN, Rosenberg H, Fletcher JE, et al. Malignant hyperthermia susceptibility in neuroleptic malignant syn-

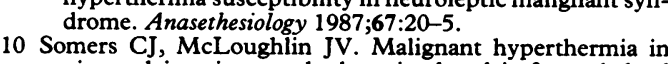
pigs: calcium ion uptake by mitochondria from skeletal muscle in susceptible animals given neuroleptic drugs and halothane. J Comp Pathol 1982;92:191-8.

11 Schiller HH, Mair WGP. Ultrastructural changes of muscle in malignant hyperthermia. J Neurol Sci 1974;21:93-100.

12 Ellis KO, Castellion AW, Honkomp LJ, et al. Dantrolene, a direct acting muscle relaxant. $J$ Pharm Sci 1973; 62:948-50.

13 Goulon M, de Rohan-Cabot P, Elkharrat D, et al. Beneficial effects of dantrolene in the treatment of neuroleptic malignant syndrome: a report of two cases. Neurology (NY) 1983;33:516-18.

14 Tolleffson GD, Garvey $M J$. The neuroleptic malignant syndrome and central dopamine metabolites. J Clin Psychopharm 1984;4:150-3.

15 Mueller PS, Vester JW, Fermaglich J. Neuroleptic malignant syndrome: successful treatment with bromocriptine. JAMA 1983;249:386-8. 